\title{
DISC1 Regulates Neurotrophin-Induced Axon Elongation via Interaction with Grb2
}

\author{
Tomoyasu Shinoda, ${ }^{1}$ Shinichiro Taya, ${ }^{1}$ Daisuke Tsuboi, ${ }^{1}$ Takao Hikita, ${ }^{1}$ Reiko Matsuzawa, ${ }^{1}$ Setsuko Kuroda, ${ }^{1}$ \\ Akihiro Iwamatsu, ${ }^{2}$ and Kozo Kaibuchi ${ }^{1}$ \\ ${ }^{1}$ Department of Cell Pharmacology, Graduate School of Medicine, Nagoya University, Showa, Nagoya 466-8550, Japan, and 2Protein Research Network, \\ Yokohama 236-0004, Japan
}

\begin{abstract}
Disrupted-in-Schizophrenia-1 (DISC1) is a candidate gene for susceptibility of schizophrenia. In the accompanying paper (Taya et al., 2006), we report that DISC1 acts as a linker between Kinesin-1 and DISC1-interacting molecules, such as NudE-like, lissencephaly-1, and 14-3-3 $\varepsilon$. Here we identified growth factor receptor bound protein 2 (Grb2) as a novel DISC1-interacting molecule. Grb2 acts as an adaptor molecule that links receptor tyrosine kinases and the Ras-extracellular signal-regulated kinase (ERK) pathway. DISC1 formed a ternary complex with Grb2 and kinesin heavy chain KIF5A of Kinesin-1. In cultured rat hippocampal neurons, both DISC1 and Grb2 partially colocalized at the distal part of axons. Knockdown of DISC1 or kinesin light chains of Kinesin-1 by RNA interference inhibited the accumulation of Grb2 from the distal part of axons. Knockdown of DISC1 also inhibited the neurotrophin-3 (NT-3)-induced phosphorylation of ERK-1/2 at the distal part of axons and inhibited NT-3-induced axon elongation. These results suggest that DISC1 is required for NT-3-induced axon elongation and ERK activation at the distal part of axons by recruiting Grb2 to axonal tips.
\end{abstract}

Key words: DISC1; neurotrophin; Grb2; axon elongation; ERK; schizophrenia

\section{Introduction}

Schizophrenia is a severe psychiatric disorder with lifelong disability. Although the causes of schizophrenia remain essentially unknown, it has been widely reported that schizophrenia has high inheritance, indicating the existence of genetic risk factors (Owen et al., 2004; Craddock et al., 2005; Harrison and Weinberger, 2005).

Disrupted-in-Schizophrenia-1 (DISC1) was originally identified as the sole gene at the breakpoint of a balanced (1:11) (q42.1; q14.3) chromosomal translocation that associated with a high inheritance of schizophrenia and other psychiatric illnesses in a large Scottish family (Millar et al., 2000; Blackwood et al., 2001). Recently, haplotype transmission analysis also revealed the implication of DISC1 in schizophrenia (Hennah et al., 2003; Ekelund et al., 2004; Hodgkinson et al., 2004; Callicott et al., 2005). In the accompanying paper (Taya et al., 2006), we reported that DISC1 directly binds to Kinesin-1, a motor protein on microtubules involved in anterograde transport in axons (Vale, 2003; Guzik and Goldstein, 2004; Hirokawa and Takemura, 2004), and regulates the

Received Sept. 2, 2006; revised 0ct. 31, 2006; accepted Nov. 1, 2006.

This work was supported by grants-in-aid for scientific research from the Ministry of Education, Culture, Sports, Science, and Technology of Japan, Special Coordination Funds for Promoting Science and Technology, the Pharmaceuticals and Medical Devices Agency, Research Fellowships from the Japan Society for the Promotion of Science for Young Scientists, the 21st Century Centre of Excellence Program from the Ministry of Education, Culture, Sports, Science, and Technology of Japan, and Research Grant 15A-2 for Nervous and Mental Disorders from the Ministry of Health, Labour, and Welfare. We thank Dr. T. Nagase and Dr. T. Takenawa for kindly providing cDNAs, Dr. N. Ozaki for his helpful discussions, Dr. A. Sawa for a helpful discussion, Dr. K. Matsumoto for kindly providing cDNAs and for his helpful discussion, and M. Goto and T. Ishii for secretarial assistance.

Correspondence should be addressed to Dr. Kozo Kaibuchi, Department of Cell Pharmacology, Graduate School of Medicine, Nagoya University, 65 Tsurumai, Showa, Nagoya 466-8550, Japan. E-mail: kaibuchi@med.nagoya-u.ac.jp. D0I:10.1523/JNEUROSCI.3825-06.2007

Copyright $\odot 2007$ Society for Neuroscience $\quad$ 0270-6474/07/270004-11\$15.00/0 transport of the NudE-like (NUDEL)/lissencephaly-1 (LIS1)/143-3 $\varepsilon$ protein complex to the distal parts of axons, suggesting that DISC1 acts as a cargo receptor that links Kinesin-1 and cargoes. The function of DISC1 as a cargo receptor, however, still remains essentially unknown.

Here we identified growth factor receptor bound protein 2 (Grb2) as a novel DISC1-interacting molecule. Grb2 is widely known as an adaptor molecule that mediates protein-protein interactions (Lowenstein et al., 1992; Takenawa et al., 1998; Buday, 1999). In neurons, Grb2 is thought to link intracellular signaling cascades and activated receptor tyrosine kinases, such as Trk receptors, a receptor family of neurotrophins (Borrello et al., 1994; Nimnual et al., 1998; MacDonald et al., 2000). Neurotrophins are important regulators of neural survival, development, function, and plasticity (Huang and Reichardt, 2001, 2003; Chao, 2003). Four neurotrophins, nerve growth factor (NGF), brainderived neurotrophic factor (BDNF), neurotrophin-3 (NT-3), and NT-4/5, have been isolated in mammals (Huang and Reichardt, 2001). Binding of neurotrophins to Trk receptors results in their dimerization and the autophosphorylation of the tyrosine residues in their cytoplasmic regions. The phosphorylated tyrosine residues recruit several adaptor proteins, including Grb2, and thereby link Trk receptors and intracellular signaling cascades, including the phosphatidylinositol-3-kinase-Akt pathway, the phospholipase C- $\gamma_{1}$ pathway, or the Ras-extracellular signal-regulated kinase (ERK) pathway (Huang and Reichardt, 2001, 2003; Chao, 2003). NGF interacts with a member of Trk receptors, TrkA, at axon terminals to produce local signals within axon terminals and retrograde signals to the neuronal cell body (Campenot and Maclnnis, 2004), suggesting that Trk receptors and their downstream signaling components localize and func- 

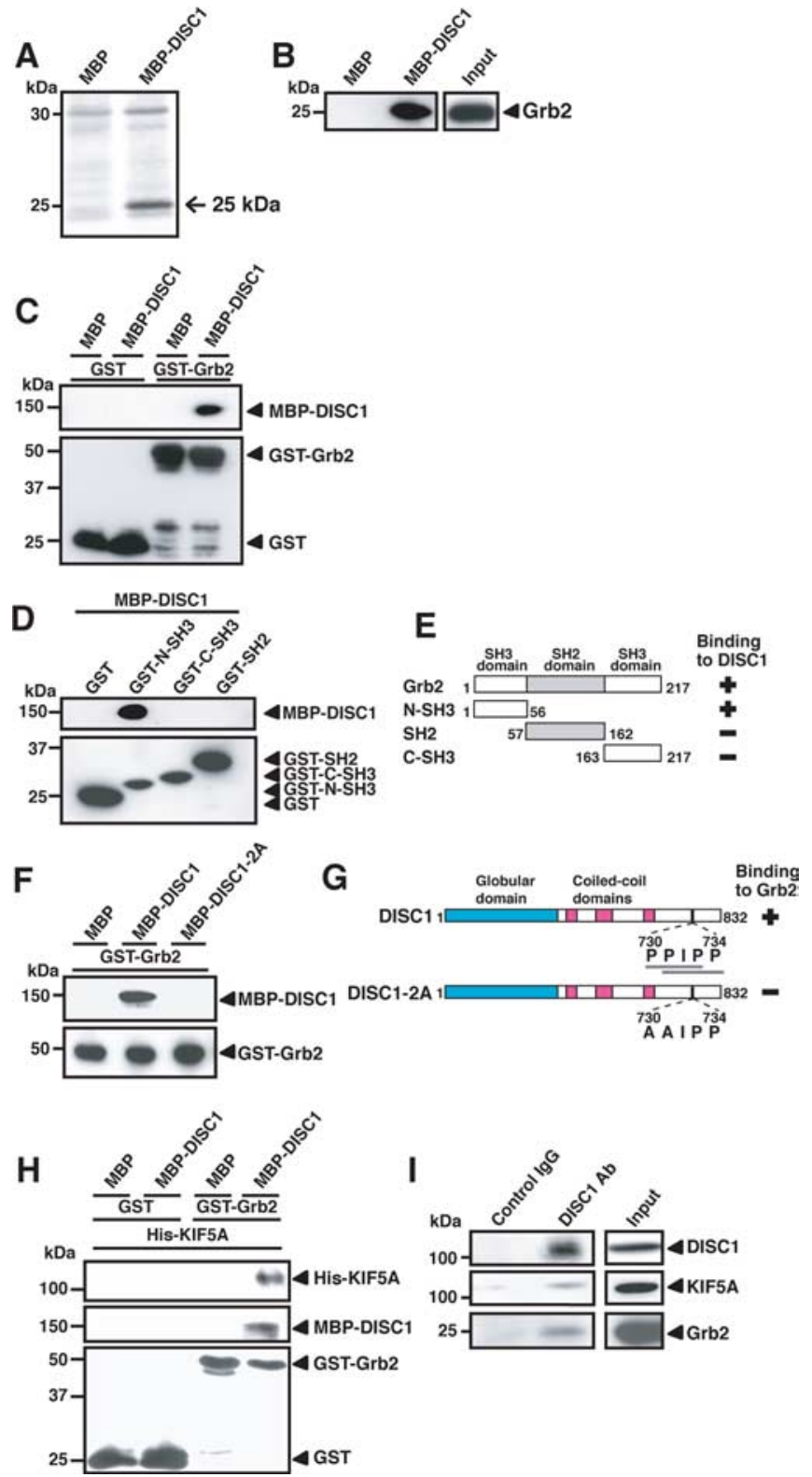

Figure 1. Identification of Grb2 as a DISC1-interacting molecule. $A$, Rat brain membrane extract was loaded onto an amylase-resin affinity column on which MBP or MBP-DISC1 was immobilized. The proteins bound to the columns were eluted by addition of the elution buffer containing $500 \mathrm{~mm} \mathrm{NaCl}$. Samples were subjected to SDS-PAGE and analyzed by the silverstaining method. A protein with apparent molecular mass of $\sim 25 \mathrm{kDa}$ (arrow) was specifically detected in the eluate from the MBP-DISC1-immobilized affinity column. $\boldsymbol{B}$, The eluate from each column was analyzed by immunoblotting with the antibody against Grb2. C, An in vitro binding assay was performed using recombinant proteins purified from E. coli. GST or GST-Grb2 was incubated with MBP or MBP-DISC1. Proteins were collected with glutathione-Sepharose beads and subjected to immunoblot analysis using anti-GST and anti-MBP antibodies. Data are representative of three independent experiments. D, GST, GST-N-SH3, GST-C-SH3, or GSTSH2 was incubated with MBP-DISC1. Proteins were collected with glutathione-Sepharose beads and subjected to immunoblot analysis using anti-GST and anti-MBP antibodies. Data are representative of three independent experiments. $\boldsymbol{E}$, Three Grb2 deletion mutants generated for the in vitro binding assay are indicated. The numbers denote amino acid positions. $F$, MBP, MBP-DISC1, or MBP-DISC1-2A was incubated with GST-Grb2. Proteins were collected with glutathione-Sepharose beads and subjected to immunoblot analysis with anti-MBP and antiGST antibodies. Data are representative of three independent experiments. G, Potential SH3 recognition motifs in DISC1 are indicated. The numbers denote amino acid positions. The minimum consensus sequence of the SH3-binding motif is PXXP ( $X$ represents any amino acid). The gray bars indicate potential SH3-binding motifs. In DISC1-2A, two proline residues (amino acid 730 and 731, respectively) were exchanged to alanines. $\boldsymbol{H}$, Purified His-KIF5A, MBP, MBPDISC1, GST, and GST-Grb2 were mixed and incubated in the indicated combinations. Proteins tion at the distal part of axons as well as at the cell body. The requirement of Grb2 for the signaling cascades downstream of Trk receptors in the physiological condition and the mechanism of proper distribution of Grb2 in neuronal cells, however, still remain to be elucidated.

In this study, we report that DISC1 forms a ternary complex with Grb2 and Kinesin-1 and that DISC1 regulates the transport of Grb2 to the distal parts of axons in a Kinesin-1-dependent manner. Furthermore, the transported Grb2 by DISC1 is involved in NT-3-induced axonal elongation.

\section{Materials and Methods}

Materials and chemicals. The expression plasmids encoding DISC1 were constructed as described in the accompanying paper (Taya et al., 2006). cDNA fragment encoding DISC1-2A was generated with a site-directed mutagenesis kit (Stratagene, La Jolla, CA) and was inserted into pMAL-C2 (New England Biolabs, Beverly, MA) or pEGFP-N3 (Clontech, Palo Alto, CA). The cDNA fragments encoding Grb2 (1-217), Grb2-N-Src homology 3 (SH3) (1-56), Grb2-SH2 (57-162), or Grb2C-SH3 (163-217) were subcloned into pGEX-4T-2 (Amersham Biosciences, Buckinghamshire, UK). The cDNA fragment encoding Grb2 (1-217) was also inserted into pEGFP-C1 (Clontech). The cDNA fragment of kinesin heavy chain KIF5A-full (1-1027) was inserted into pRSET-C1 (Invitrogen, Carlsbad, CA). The other expression plasmid encoding myc-KIF5A-full or myc-KIF5A-headless (KIF5A-HL) was prepared as described in the accompanying paper (Taya et al., 2006). cDNA encoding rat Grb2 was kindly provided by Dr. Takenawa (University of Tokyo, Tokyo, Japan). pEGFP-ERK-2 was kindly provided by Dr. Matsumoto (Nagoya University, Nagoya, Japan). Antibodies against Grb2 (MBL, Aichi, Japan), GFP (MBL), KIF5A (Chemicon, Temecula, CA), $\beta$-tubulin (Sigma, St. Louis, MO), Tau-1 (Chemicon), TUJ-1 (Covance Research Products, Denver, PA), c-Myc (Santa Cruz Biotechnology, Santa Cruz, CA), and phospho-ERK-1/2 (Cell Signaling Technology, Beverly, MA) were purchased. Polyclonal antibody against DISC1 was prepared as described in the accompanying paper (Taya et al., 2006). Polyclonal antibody against kinesin light chains (KLCs) was prepared as described previously (Kimura et al., 2005). Celltracker Orange 5-(and-6)(((4-chloromethyl)benzoyl)-amino)tetramethylrhodamine (CMTMR) and Green chloromethyl-fluorescein-diacetate were purchased from Invitrogen. Recombinant NGF (Upstate Biotechnology, Charlottesville, VA) and NT-3 (Peprotech, London, UK) were purchased. Mitogen-activated protein kinase kinase 1/2 (MEK-1/2) inhibitors PD98059 [2-(2-amino-3-methyoxyphenyl)-4H-1-benzopyran-4-one] and U0126 [1,4-diamino-2,3-dicyano1,4-bis(o-aminophenylmercapto)butadiene] were obtained from Wako (Osaka, Japan).

Affinity column chromatography and mass spectral analysis. Rat brain membrane extract was loaded onto beads coated with maltose-binding protein (MBP) or MBP-DISC1. The proteins bound to the affinity columns were eluted by the addition of buffer containing $500 \mathrm{~mm} \mathrm{NaCl}$. The eluates were subjected to SDS-PAGE, and the bound proteins were evaluated by silver staining. We identified proteins by comparing the molecular weights determined by matrix-assisted laser desorption/ionization time-of-flight mass spectrometry and theoretical peptide masses from the proteins registered in NCBInr (National Center for Biotechnology Information, Bethesda, MD).

In vitro binding assay. Glutathione $S$-transferase (GST)-, MBP-, and His-tagged recombinant proteins were expressed in Escherichia coli BL21(DE3) and purified by glutathione-Sepharose 4B beads (Amersham Biosciences), amylase-resin beads (New England Biolabs), and Ni-NTA agarose (Qiagen, Hilden, Germany), respectively, according to the pro-

$\leftarrow$

were then collected with glutathione-Sepharose beads and subjected to immunoblot analysis with anti-His, anti-MBP, and anti-GST antibodies. Data are representative of three independent experiments. I, Endogenous DISC1 was immunoprecipitated by anti-DISC1 antibody from rat brain lysate. Immunoprecipitated samples were subjected to immunoblot analysis with antiDISC1, Grb2, and KIF5A antibodies. Data are representative of three independent experiments. 
tocols of the manufacturers. Proteins were incubated in buffer (20 mm Tris, $1 \mathrm{~mm}$ EDTA, and $1 \mathrm{~mm}$ DTT) with glutathione-Sepharose 4B beads for $1 \mathrm{~h}$. Bound proteins were eluted by the addition of $10 \mathrm{~mm}$ glutathione. Portions of the eluates were subjected to SDS-PAGE, followed by immunoblot analysis with antibodies against GST, MBP, or His.

GST pull-down assay. COS7 cells were transfected with the expression vectors by Lipofectamine (Invitrogen) and cultured for $48 \mathrm{~h}$. Cells were lysed with buffer [ $20 \mathrm{~mm}$ Tris- $\mathrm{HCl}, 1$ mм EDTA, $150 \mathrm{~mm} \mathrm{NaCl}, 1 \mathrm{~mm}$ DTT, $0.1 \%$ (w/v) Triton X-100, $10 \mu \mathrm{M} p$-amidinophenyl methanesulfonyl fluoride hydrochloride, and $10 \mu \mathrm{g} / \mathrm{ml}$ leupeptin]. The lysate was sonicated and then clarified by centrifugation at $12,000 \times$ $g$ for $10 \mathrm{~min}$ at $4^{\circ} \mathrm{C}$. The soluble supernatant was incubated with glutathione-Sepharose $4 \mathrm{~B}$ beads. Portions of the eluates were subjected to SDS-PAGE, followed by immunoblot analysis with the indicated antibodies.

Immunoprecipitation analysis. Adult rat brain lysates were prepared with lysis buffer $(20$ mм Tris, 1 mм EDTA, $150 \mathrm{~mm} \mathrm{NaCl}$, and 1\% NP-40, pH 7.5). An immunoprecipitation assay was performed with various antibodies and protein G Sepharose (Amersham Biosciences). The precipitated proteins were eluted with SDS sample buffer and subjected to immunoblot analysis.

Small interference RNAs. A 21-oligonucleotide small interference RNA (siRNA) duplex was synthesized to target the rat Grb2 sequence $5^{\prime}$ CATGTTTCCCCGCAATTAT-3' (Jiang et al., 2003). siRNAs against rat DISC1, KLC1, and KLC2 were synthesized and used as described in the accompanying paper (Taya et al., 2006). A scramble sequence, 5'-CAGTCGCGTTTGCGACTGG-3', which had no homology to any mammalian mRNAs, was used as a negative control.

Culture of PC12 cells. PC12 cells were cultured in DMEM containing $5 \%$ horse serum and $5 \%$ fetal bovine serum. Transfections of cells with plasmids or siRNAs were performed with Lipofectamine 2000 (Invitrogen). For the ERK phosphorylation assay (see Fig. 8), cells were grown in DMEM with 5\% horse serum and 5\% fetal bovine serum for $1 \mathrm{~d}$ after transfection and were then cultured in DMEM without serum for $1 \mathrm{~d}$. After the treatment with NGF at a final concentration of $100 \mathrm{ng} / \mathrm{ml}$, cells were treated with $10 \%(\mathrm{w} / \mathrm{v})$ trichloroacetic acid. The resulting cell precipitates were subjected to SDS-PAGE and immunoblot analysis.

Preparation and cultures of hippocampal neurons. Hippocampal neurons were prepared from embryonic day 18 rat embryos and cultured as described in the accompanying paper (Taya et al., 2006). Transfections of neurons were performed by the calcium phosphate method, as described previously (Inagaki et al., 2001), or by Nucleofector (Amaxa, Cologne, Germany).

Immunofluorescence analysis. Axons were determined by the staining of Tau-1 and its morphology (Craig and Banker, 1994). The accumulation of Grb2 at the distal part of axons was quantified as described in the accompanying paper (Taya et al., 2006). In brief, the fluorescence intensity of immunostained Grb2 was measured at both the central region of the growth cone and the intermediate region of the axonal shaft between the cell body and the distal part of the axon. The accumulation of Grb2 was scored as the percentage of cells with $>150 \%$ fluorescence intensity of immunostained Grb2 at the growth cones against those at the axonal shaft. For, NT-3 stimulation assays, transfected neurons were cultured with the medium described above, supplemented additionally with NT-3 at a concentration of $100 \mathrm{ng} / \mathrm{ml}$. To obtain the fluorescence intensity of immunostained phospho-ERK-1/2, neurons were labeled with Celltracker Orange CMTMR according to the protocol of the manufacturer.
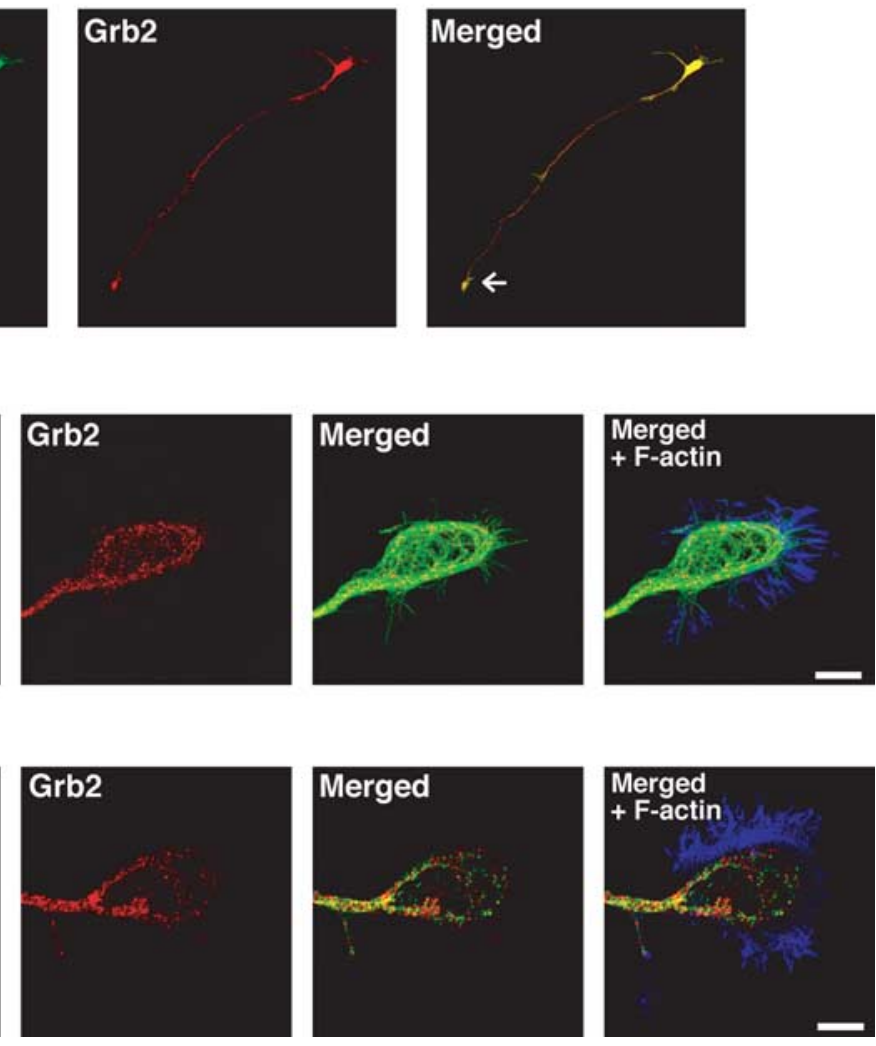

Colocalization of DISC1 and Grb2 in neuron. $A$, Rat primary hippocampal neurons were fixed at DIV3 and immunocones of a rat hippocampal neuron showed the staining of tubulin and Grb2 (B) and DISC1 and Grb2 (C). The peripheral regions of the growth cones are visualized by the staining of F-actin with Alexa 647-conjugated phalloidin. Scale bars, $10 \mu \mathrm{m}$.

The mean fluorescence intensity of phospho-ERK-1/2 at cell body or growth cone was determined by dividing the sum of all pixel intensities in regions of interest (cell body) or central growth cone area (growth cone) by those of CMTMR. In some experiments, neurons were cultured with MEK-1/2 inhibitor PD98059 or U0126 at a final concentration of 20 or 1 $\mu \mathrm{M}$, respectively. Neurons were fixed and immunostained as described in the accompanying paper (Taya et al., 2006) and observed with a laser confocal microscope (model LSM510 PASCAL; Zeiss, Oberkochen, Germany).

Time-lapse observation. Hippocampal neurons were seeded on $3.5 \mathrm{~cm}$ glass-bottom dish and then transfected with expression plasmid encoding enhanced green fluorescent protein (EGFP) Grb2 at $1 \mathrm{~d}$ in vitro (DIV1) and with cyanine 3 (Cy3)-scramble, Cy3-rDISC1-siRNAs, or Cy3-KLCs-siRNAs at DIV2. At DIV4, the areas of distal part of axons in Cy3- and EGFP-positive neurons were photobleached with 75 laser irradiations and then observed at $37^{\circ} \mathrm{C}$ for $4 \mathrm{~min}$ in a microincubator (Sankei, Tokyo, Japan) using a laser scanning confocal microscope equipped with Zeiss Axiovert $100 \mathrm{M}$ (Plan Apochromat $63 \times / 1.40 \mathrm{nu}-$ merical aperture oil immersion objective).

\section{Results}

Identification of Grb2 as a DISC1-interacting molecule

To identify novel DISC1-interacting molecules, we performed an affinity chromatography using human DISC1 as a bait. In the accompanying paper (Taya et al., 2006), we identified DISC1interacting molecules, such as NUDEL, LIS1, 14-3-3e, and Kinesin-1, from the cytosol extract derived from rat brain. This time, we screened DISC1-binding candidates from the rat brain membrane extract. The extract was loaded onto an amylose-resin affinity column on which MBP alone or MBP-DISC1 was immobilized. The proteins bound to the columns were eluted by addi- 

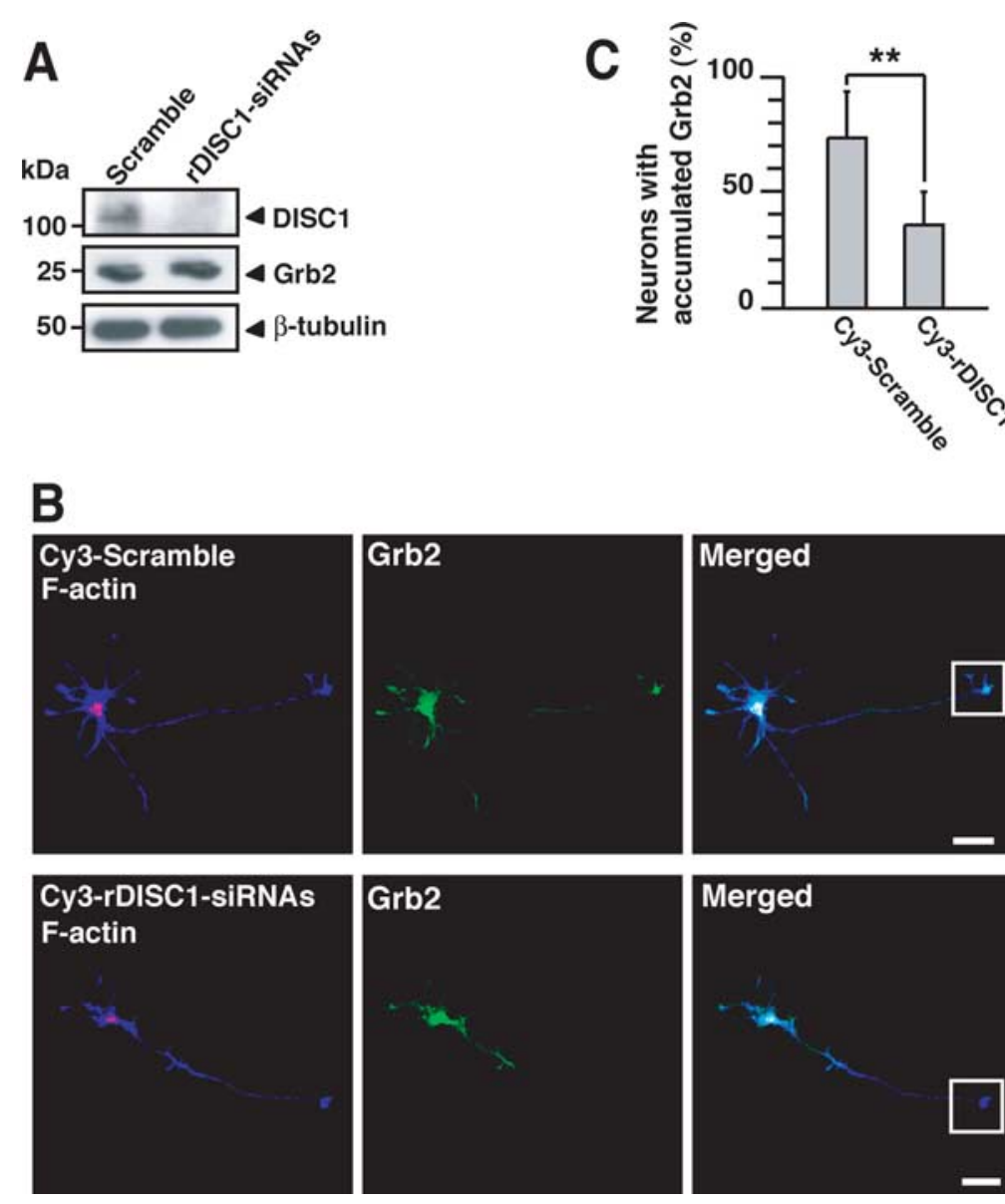

Figure 3. Regulation of Grb2 localization by DISC1. A, PC12 cells were transfected with scramble or rDISC1-siRNAs and then differentiated with NGF for $48 \mathrm{~h}$. Cell lysates were subjected to immunoblot analysis with antibodies against DISC1, Grb2, and $\beta$-tubulin. B, Hippocampal neurons were transfected with Cy3-scramble or Cy3-rDISC1-siRNAs at DIV1. Cells were fixed at DIV3 and then stained with anti-Grb2 antibody and Alexa 647-conjugated phalloidin. The transfected cells were identified by the fluorescence of $\mathrm{C} y 3$ at the cell body. The enlarged images of the distal part of axons are shown. Scale bars, $20 \mu \mathrm{m}$. C, Quantitative analysis of Grb2 accumulation. The accumulation of Grb2 at the distal part of axons was assessed (see Materials and Methods). Numbers of cells used for each calculation are $>50$, and data are means \pm SE of three independent experiments (Student's $t$ test, $\left.{ }^{* *} p<0.01\right)$.

tion of the $500 \mathrm{~mm} \mathrm{NaCl}$-containing buffer. Several proteins with apparent molecular mass of $\sim 150,115,50,37$ (data not shown), and $25 \mathrm{kDa}$ (Fig. $1 \mathrm{~A}$ ) were specifically detected in the eluate from the MBP-DISC1-immobilized affinity column. The 150, 50, and $37 \mathrm{kDa}$ proteins were full-length or degradation products of MBP-DISC1. Mass spectral analysis revealed that the $25 \mathrm{kDa}$ protein was identical to rat Grb2. The $115 \mathrm{kDa}$ protein was not identified. The immunoblot analysis with anti-Grb2 antibody revealed an interaction between DISC1 and Grb2 (Fig. $1 B$ ). Grb2 acts as an adaptor molecule that mediates protein-protein interactions. Grb2 is composed of two SH3 domains and one $\mathrm{SH} 2$ domain. The SH3 domains of Grb2 interact with a number of intracellular proteins, including son of sevenless (Sos) and Grb2associated binding protein 1 (Gab1) (Takenawa et al., 1998; Buday, 1999). The SH2 domain of Grb2, conversely, recognizes phosphotyrosine motifs, especially those of activated receptor tyrosine kinases. To confirm the direct interaction between DISC1 and Grb2, we performed an in vitro binding assay using purified recombinant proteins. MBP-DISC1 interacted with GST-Grb2 but not with GST alone (Fig. 1C). These results indicate that DISC1 directly interacts with Grb2.

Next, we examined which domains of Grb2 are capable of binding to DISC1. GST-N-SH3 interacted with MBP-DISC1, whereas either GSTSH2 or GST-C-SH3 did not (Fig. $1 D, E$ ), indicating that the N-terminal $\mathrm{SH} 3$ domain of Grb2 is sufficient for binding to DISC1. SH3 domains recognize prolinerich motifs, with the minimal consensus sequence $\mathrm{PXXP}(X$ represents any amino acid) (Pawson, 1993). Human DISC1 contains two putative SH3-binding motifs at amino acid residues $730-733$ and 731-734 (Fig. $1 G$ ), which are overlapped with each other and conserved in mouse and rat DISC1. Because it was reported that the substitution of prolines in the proline-rich motif by alanines resulted in its defective binding to $\mathrm{SH} 3$ domain (Rozakis-Adcock et al., 1993), we generated a DISC1 mutant, named DISC1-2A, that had amino acid substitution by alanines in the first prolines in both proline-rich motifs (Pro730 and Pro-731) (Fig. 1G). To examine whether the proline-rich motifs are recognized by Grb2, an in vitro binding assay using recombinant wild-type DISC1 and DISC1-2A proteins was performed. MBPDISC1 interacted with GST-Grb2, whereas MBP alone and MBP-DISC1-2A did not (Fig. 1F), indicating that the proline-rich motifs in DISC1 are responsible for the direct interaction with Grb2. Because the proline-rich motifs in DISC1 are closely located to the NUDEL-binding regions (amino acids 598-697 and 795854) (Morris et al., 2003; Ozeki et al., 2003), we examined whether Grb2 and NUDEL simultaneously form a complex with DISC1. COS7 cells were transfected with the expression vectors encoding GST-Grb2, myc-DISC1, and NUDELGFP. When GST-Grb2 was precipitated, myc-DISC1 was co-precipitated (supplemental Fig. 1, lanes 3-5, available at www.jneurosci.org as supplemental material). In this condition, the co-precipitation of NUDEL-GFP was not observed (data not shown). When NUDEL-GFP was overexpressed, the amount of myc-DISC1 coprecipitated with GSTGrb2 was slightly decreased (supplemental Fig. 1, lane 5, available at www.jneurosci.org as supplemental material). These results suggest that the ternary complex formation between NUDEL, DISC1, and Grb2 does not occur in COS7 cells.

\section{Ternary complex formation between Grb2, DISC1, and KIF5A}

In the accompanying paper (Taya et al., 2006), we reported that the amino acid residues 341-400 of DISC1 directly interacted with a kinesin heavy chain, KIF5A, which is a component of Kinesin-1. This raised a possibility that DISC1, Grb2, and Kinesin-1 form a ternary complex. To examine whether DISC1 forms a ternary complex with Grb2 and KIF5A, we performed an in vitro binding assay using purified recombinant proteins. GSTGrb2 interacted with His-KIF5A in the presence of MBP-DISC1, whereas GST-Grb2 did not interact with His-KIF5A in the absence of MBP-DISC1 (Fig. $1 H$ ), suggesting that the formation of 
the ternary complex depends on DISC1. We further examined the physiological interaction of these molecules by immunoprecipitation. Endogenous DISC1 was precipitated from adult rat brain lysate with anti-DISC1 antibody. Grb2 as well as KIF5A were coprecipitated with DISC1 (Fig. 1I). We also performed immunoprecipitation using anti-Grb2 antibody. AntiGrb2 antibody precipitated endogenous Grb2, whereas DISC1 and KIF5A were not coprecipitated (data not shown), possibly because of low precipitation efficiency of the antibody or low expression level of either Grb2 or DISC1. Together, these results suggest that Grb2, DISC1, and KIF5A make a ternary complex under physiological conditions.

\section{Regulation of Grb2 localization by DISC1}

To investigate possible roles of DISC1 in Grb2 distribution, we examined the intracellular localizations of DISC1 and Grb2 in cultured rat hippocampal neurons. In DIV3 neurons, the immunoreactivity of Grb2 was observed in the cell body, minor processes, and the distal part of axon (Fig. $2 \mathrm{~A}$ ). We further investigated the localization of DISC1 and Grb2 in axonal growth cones. On the basis of the cytoskeletal structures, the area of the axonal growth cone is classified into peripheral, transition, and central regions (Dent and Gertler, 2003). In the accompanying paper (Taya et al., 2006), we showed that DISC1 localized in the central region of growth cones along microtubules. Highmagnification views of the growth cones revealed that Grb2 also localized along microtubules in the central region (Fig. $2 B, C)$ and partially colocalized with DISC1 (Fig. 2C).

Next, we investigated whether DISC1 regulates Grb2 localization by knockdown of DISC1 by RNA interference. Because the expression level of DISC1 in a cultured rat hippocampal neuron is low, we used PC12 cells for biochemical assays. The transfection of rDISC1-siRNAs resulted in decreased expression level of DISC1, which did not affect the expression levels of KLC1, KLC2, KIF5A, $\beta$-tubulin (Taya et al., 2006), and Grb2 (Fig. 3A). Similar results were obtained when using Cy3-rDISC1-siRNAs (data not shown). We transfected Cy3-scramble or Cy3-rDISC1-siRNAs into cultured hippocampal neurons. In the neurons transfected with Cy3-scramble, the accumulation of Grb2 at the distal part of axons were observed, whereas the accumulation of Grb2 was inhibited in the neurons transfected with Cy3-rDISC1-siRNAs (Fig. 3 B, C). Transfection of Cy3-rDISC1-siRNAs did not alter the shape of growth cones, as determined by the staining of filamentous actin (F-actin) (Fig. $3 B)$. Similar results were obtained when using nonlabeled

\section{B}
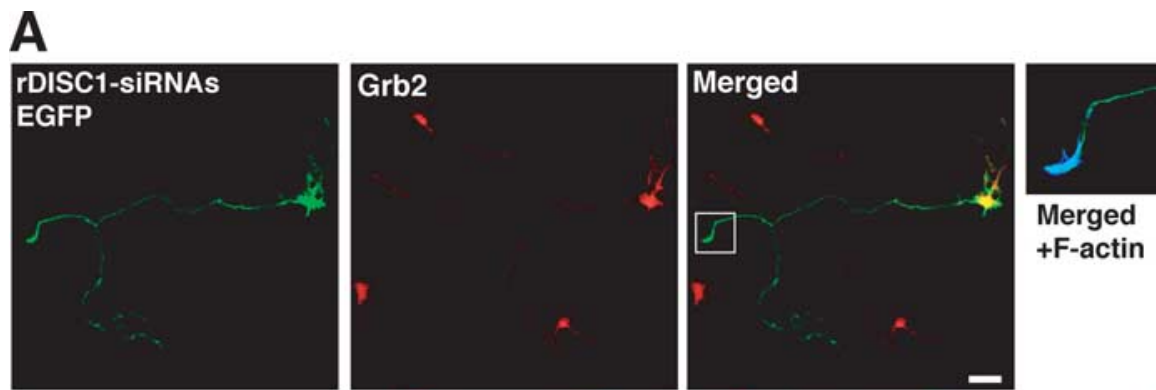

Merged $+\mathrm{F}$-actin
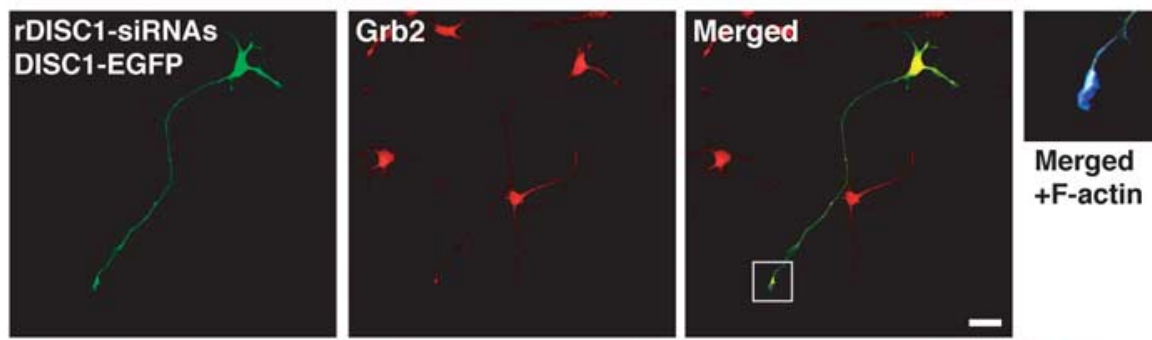

Merged + F-actin
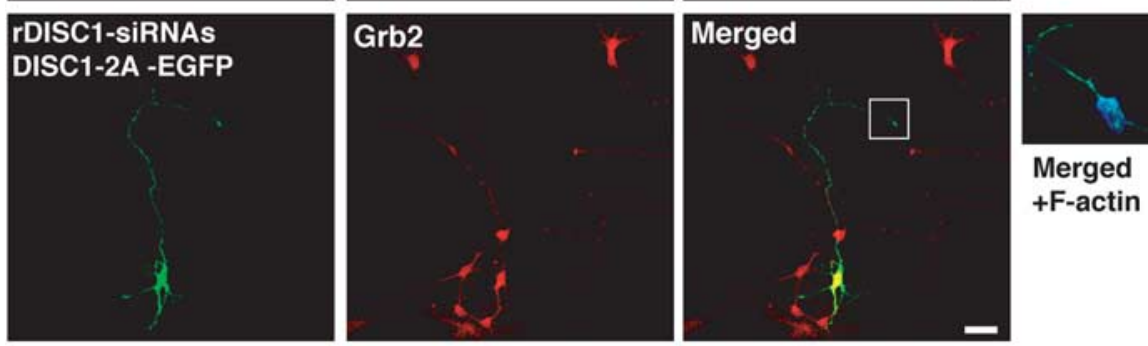

Merged $+\mathrm{F}$-actin

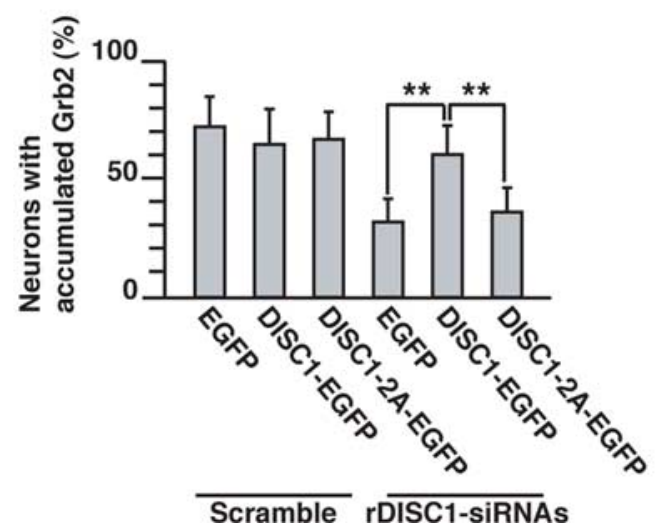

Figure 4. Rescue experiment of the defective localization of Grb2 by DISC1 knockdown. $\boldsymbol{A}$, Hippocampal neurons were cotransfected with rDISC1-siRNAs and the expression plasmid encoding EGFP, DISC1-EGFP, or DISC1-2A-EGFP at DIV1. Neurons were fixed at DIV3 and immunostained with antibodies against GFP and Grb2. The distal part of axons were visualized by the staining with Alexa 647-conjugated phalloidin. Enlarged images of the distal part of axons are shown. Scale bars, $20 \mu \mathrm{m}$. B, Quantitative analysis of Grb2 accumulation in the neurons cotransfected with siRNAs and expression plasmids. The accumulation of Grb2 at the distal part of axons was assessed (see Materials and Methods). Numbers of cells used for each calculation are $>50$, and data are means \pm SE of three independent experiments (Student's $t$ test, ${ }^{* *} p<0.01$ ).

rDISC1-siRNA instead of Cy3-rDISC1-siRNAs (Fig. 4A,B). These results suggest that DISC1 is involved in the distribution of Grb2 at the distal part of axons.

To confirm whether the direct interaction between Grb2 and DISC1 is essential for the accumulation of Grb2 in the distal part of axons, we performed a rescue experiment using human DISC1, which is resistant to rDISC1-siRNAs. The expression level of DISC1-EGFP or DISC1-2A-EGFP was not altered by the transfection of rDISC1-siRNAs (data not shown). Hippocampal neurons were cotransfected with rDISC1-siRNAs and the ex- 


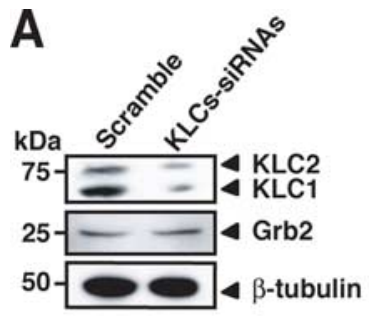

E

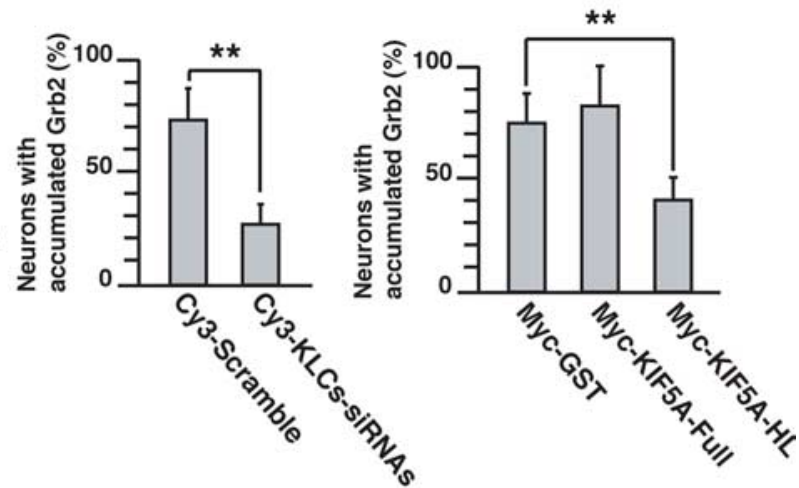

B
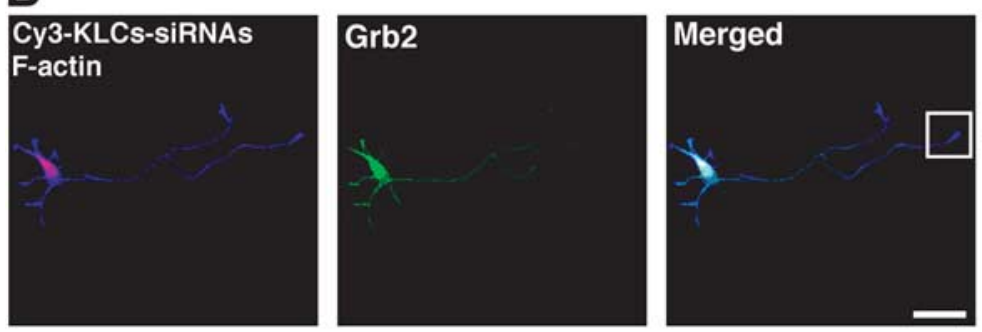

C
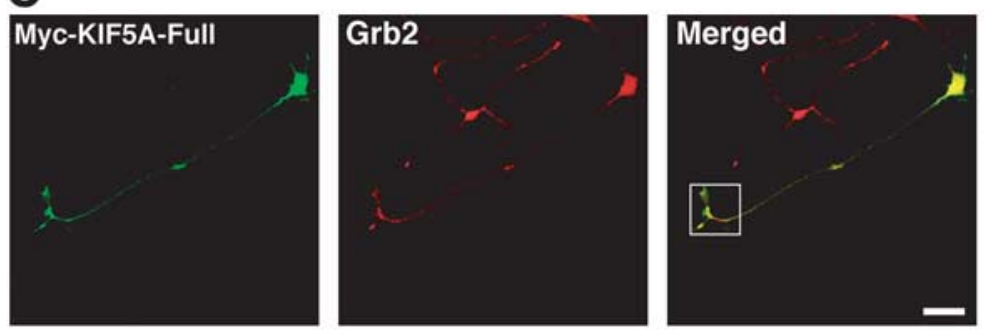

D
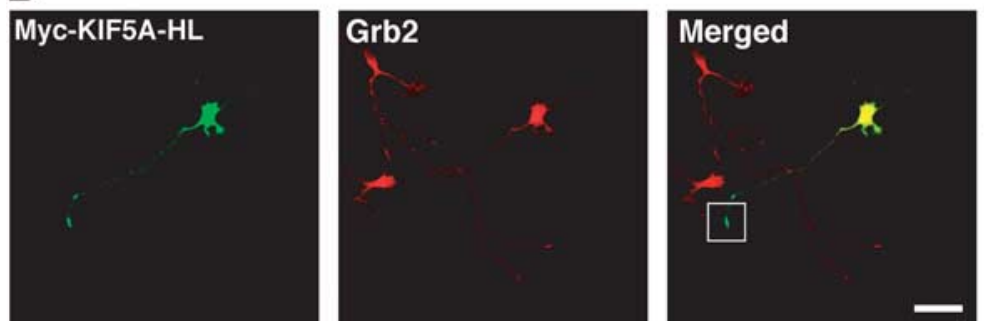

Figure 5. Regulation of DISC1/Grb2 localization by Kinesin-1.A, PC12 cells were transfected with Cy3-scramble or Cy3-KLCssiRNAs and then differentiated with NGF for $48 \mathrm{~h}$. Cell lysates were subjected to immunoblot analysis with antibodies against KLCs, Grb2, and $\beta$-tubulin. B, Hippocampal neurons were transfected with Cy3-KLCs-siRNAs at DIV1. Cells were fixed at DIV3 and stained with antibodies against Grb2. Cells were also stained by Alexa 647-conjugated phalloidin. Transfected cells were identified by the fluorescence of Cy3. Enlarged image of the distal part of the axon is shown. Scale bar, $20 \mu \mathrm{m}$. C, D, Hippocampal neurons were transfected with the expression plasmid encoding myc-KIF5A-full (C) or myc-KIF5A-HL (D) at DIV1. Cells were fixed at DIV3 and then immunostained with antibodies against myc and Grb2. The distal part of axons were visualized by staining of F-actin with Alexa 647-conjugated phalloidin. Enlarged images of the distal part of axons are shown. Scale bars, $20 \mu \mathrm{m} . \boldsymbol{E}, \boldsymbol{F}$, Quantitative analysis of Grb2 accumulation in the neurons transfected with Cy3-scramble or Cy3-KLCs-siRNAs $(\boldsymbol{E})$ or the expression plasmid encoding myc-GST, myc-KIF5A-full, or myc-KIF5A-HL $(\boldsymbol{F})$. The accumulation of Grb2 at the distal part of axons was assessed (see Materials and Methods). Numbers of cells used for each calculation are $>50$, and data are means \pm SE of three independent experiments (Student's $t$ test, ${ }^{* *} p<0.01$ ).

pression plasmid encoding EGFP, DISC1-EGFP, or DISC1-2AEGFP. In neurons expressing either EGFP or DISC1-2A-EGFP, the accumulation of Grb2 was inhibited at the distal part of axons (Fig. $4 A, B$ ). In contrast, the immunoreactivity of Grb2 at the distal part of axons was recovered in neurons expressing DISC1EGFP under the conditions in which endogenous DISC1 was
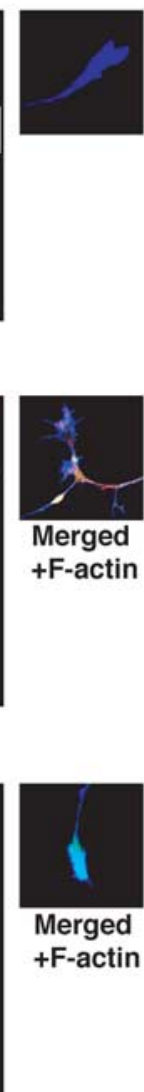

knocked down (Fig. 4A,B), indicating that the defective accumulation of Grb2 at the distal part of axons induced by the knockdown of DISC1 was rescued by wild-type DISC1 but not by DISC1-2A.

\section{Regulation of Grb2 localization \\ by Kinesin-1}

In the accompanying paper (Taya et al., 2006), we showed that the knockdown of KLCs or the expression of a mutant form of KIF5A, named KIF5A-HL, which lacks motor domain and acts as a dominantnegative form for Kinesin-1 (Nakata and Hirokawa, 2003; Nishimura et al., 2004; Kimura et al., 2005), resulted in the loss of DISC1 accumulation at the distal part of axons. We next investigated whether Grb2 distribution is regulated by Kinesin-1. Transfection of Cy3-KLCs-siRNAs resulted in decreased expression levels of both KLC1 and KLC2 (Kimura et al., 2005), whereas the expression levels of DISC1, KIF5A, $\beta$-tubulin (Taya et al., 2006), and Grb2 were not changed (Fig. 5A). In Cy3-KLCs-siRNAs-transfected neurons, the accumulation of Grb2 at the distal part of axons was decreased compared with Cy3-scramble-transfected neurons (Figs. 3B, 5B,E). We next examined the effect of KIF5A-full or KIF5A-HL on the distribution of Grb2. Expression of myc-KIF5A-full slightly increased the accumulation of Grb2 at the distal part of axons compared with those of neurons expressing myc-GST (Fig. 5C,F). Conversely, the accumulation of Grb2 was inhibited in the neurons expressing mycKIF5A-HL (Fig. 5D, F).

We further investigated the transport of Grb2 in axons by time-lapse imaging of EGFP-Grb2. Hippocampal neurons were transfected with the expression vector encoding EGFP-Grb2 at DIV1 and then transfected with Cy3-labeled siRNAs at DIV2. The immunoreactivity of EGFPGrb2 was observed in the entire neuron, including axon shafts and growth cones at DIV4, under these conditions (Fig. 6A, B). The distal parts of axons of both EGFPand Cy3-positive neurons were photobleached, and then the recovery of the fluorescence of EGFP-Grb2 was observed. The control neurons transfected with Cy3-scramble showed a steady recovery of the fluorescence in photobleached areas (Fig. $6 A, C$ ). In DISC1 or KLCs knockdown neurons, the recovery of the fluorescence of EGFP-Grb2 was clearly delayed (Fig. $6 A, C$ ). When neurons were treated with Nocodazol and placed on ice for $4 \mathrm{~min}$, the movement of EGFPGrb2 and NUDEL-GFP was prevented (data not shown) (Taya et al., 2006), suggesting that the recovery of EGFP-Grb2 depends on the transport on the rails of microtubules, because the transport 

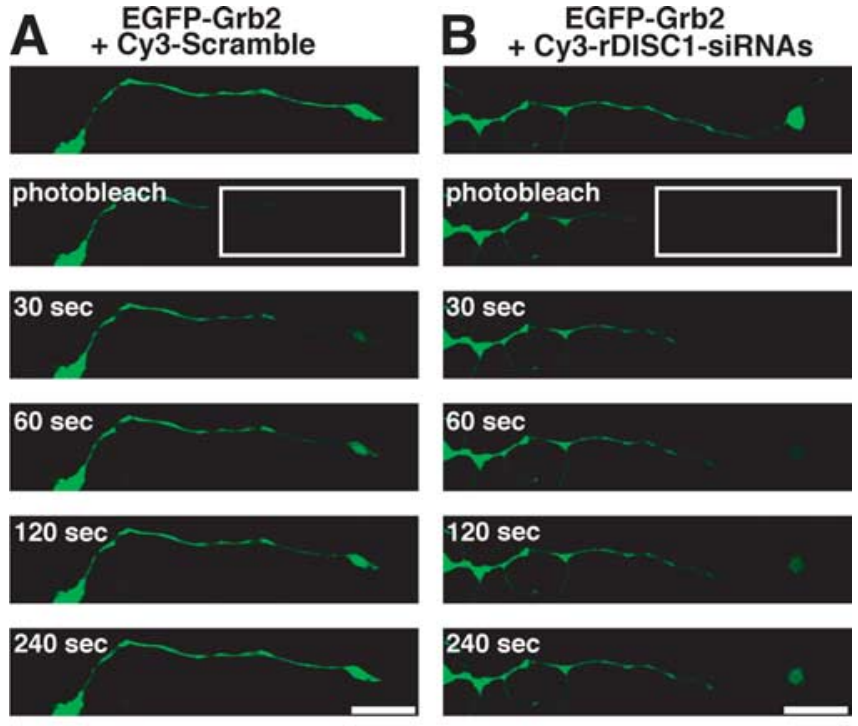

\section{$240 \mathrm{sec}$}
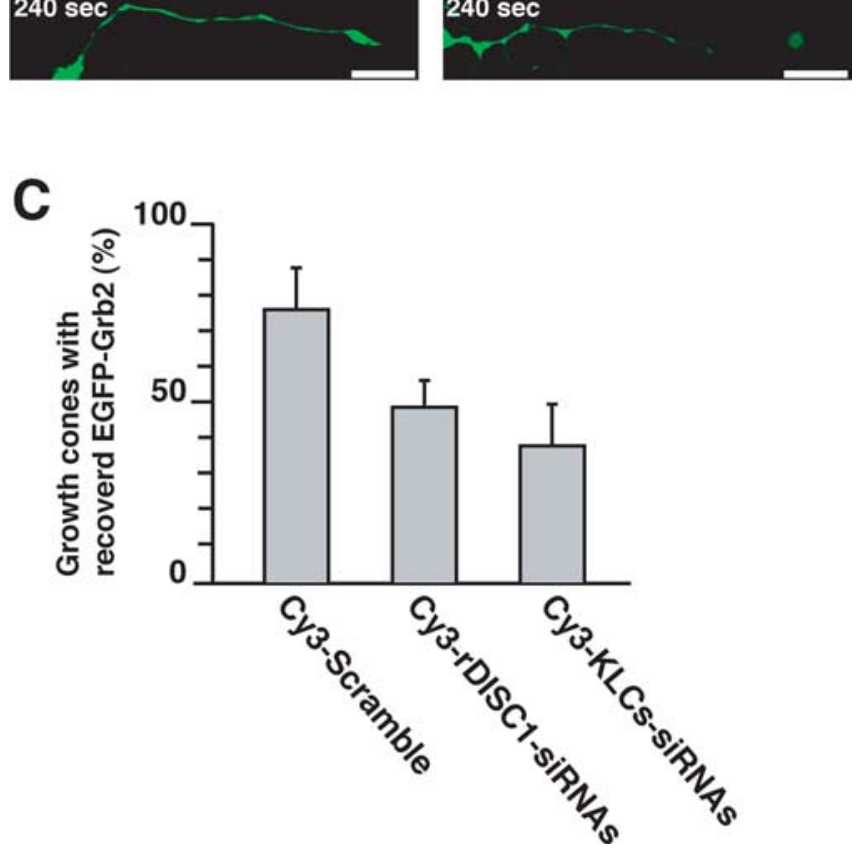

Figure 6. Effect of DISC1 or KLCS knockdown on the transport of EGFP-Grb2. $\boldsymbol{A}$, B, Hippocampal neurons were transfected with the expression plasmid encoding EGFP-Grb2 at DIV1 and then transfected with Cy3-scramble ( $\boldsymbol{A})$ or Cy3-rDISC1-siRNAs ( $\boldsymbol{B})$ at DIV2. At DIV4, the areas of the distal parts of axons of Cy3- and EGFP-positive neurons were photobleached, and the recovery of the fluorescence of EGFP-Grb2 was observed. Scale bars, $10 \mu \mathrm{m}$. C, Quantification of the recovery of the fluorescence of EGFP-Grb2 at the photobleached area in the distal parts of axons of Cy3-scramble-, Cy3-rDISC1-siRNAs-, or Cy3-KLCs-siRNAs-transfected neurons. The growth cones with $>70 \%$ fluorescent recovery in 4 min after photobleach were considered as "growth cones with recovered EGFP-Grb2." Data are means \pm SE of five independent experiments (total numbers of cells is 50 ).

along microtubules is faster than the simple diffusion and so is inhibited by microtubule disruption (Galbraith et al., 1999). Together, these results indicate that the accumulation of Grb2 at the distal part of axons is regulated by the DISC1/Kinesin-1 complex.

\section{Requirement of Grb2 in neurotrophin-induced ERK phosphorylation}

It has been reported that Grb2 associates with activated Trk receptors, a receptor family of neurotrophins, via direct interaction (MacDonald et al., 2000) or via interactions with Shc (Src homology 2 domain-containing transforming protein) (Borrello et al., 1994), Frs2 (fibroblast growth factor receptor substrate 2) (Kouhara et al., 1997), SH2-B, and rAPS (Qian et al., 1998). Grb2 recruits Sos, a guanine-nucleotide exchange factor for Ras, beside the intracellular region of receptor tyrosine kinases, thereby activating the Ras-ERK signaling pathway (Lowenstein et al., 1992; Takenawa et al., 1998; Buday, 1999). The activation of Ras or Raf is sufficient for NGF-induced axon elongation in cultured sensory neurons (Markus et al., 2002). We showed recently that Ras plays critical roles in the establishment of neuronal polarity (Yoshimura et al., 2006). It is also reported that the treatment of hippocampal neurons with neurotrophins, such as BDNF and NT-3, induces the activation of Ras and ERK (Iida et al., 2001) and enhances axon elongation (Ip et al., 1993; Labbele and Leclerc, 2000; Yoshimura et al., 2005).

To investigate the involvement of Grb2 and DISC1 in the Ras-ERK pathway, we examined the effect of the knockdown of Grb2 or DISC1 in ERK phosphorylation with NGF treatment. The transfection of rat Grb2-specific siRNA (rGrb2-siRNA) into PC12 cells effectively decreased the expression level of Grb2, as described previously (Fig. 7A) (Jiang et al., 2003). Next, PC12 cells were cotransfected with EGFP-ERK-2 and scramble rDISC1-siRNAs or rGrb2-siRNA and then treated with NGF for 5, 10, 20, or 60 min. Knockdown of DISC1 did not affect the phosphorylation levels of EGFP-ERK-2 during NGF treatment compared with those of scramble-transfected cells. The transfection of rGrb2-siRNA resulted in decreased phosphorylation levels of EGFP-ERK-2 (Fig. 7B). These results suggest that Grb2 is essential for ERK activation during NGF stimulation and that DISC1 itself is not required for the ERK activation pathway in PC12 cells under these conditions.

We next investigated the involvement of the Ras-ERK pathway in axon elongation in hippocampal neurons. The activation of ERK-1/2 requires its phosphorylation by MEK-1/2 (Errede et al., 1995). We added PD98059 or U0126, both known as specific inhibitors for MEK-1/2, to the cultured hippocampal neurons at DIV1. Treatment with PD98059 or U0126 for $2 \mathrm{~d}$ did not alter the length of axons (Fig. 7C). We next treated hippocampal neurons with PD98059 or U0126 in the presence of NT-3 instead of NGF because NGF does not alter axon length in cultured hippocampal neurons (Ip et al., 1993; Labbele and Leclerc, 2000). NT-3 induced axon elongation when cultured in the absence of MEK inhibitors (Fig. 7C). Conversely, treatment with PD98059 or U0126 blocked the NT-3-induced axon elongation (Fig. 7C). The intracellular distributions of both DISC1 and Grb2 were not altered by treatment with MEK inhibitors or NT-3 (data not shown). These results suggest that the activation of the Ras-ERK pathway is required for NT-3-induced axon elongation in cultured hippocampal neurons.

\section{Requirement of DISC1 in neurotrophin-induced axon elongation}

We next examined the involvement of DISC1 and Grb2 in the NT-3-induced ERK phosphorylation in hippocampal neurons. Hippocampal neurons were cotransfected with scramble or rDISC1-siRNAs and the expression vector encoding EGFP, and then the phosphorylation level of ERK-1/2 at cell bodies or growth cones was measured. In the neurons transfected with EGFP and scramble siRNA, the phosphorylation levels of ERK$1 / 2$ in both cell bodies and growth cones were increased by a 30 min treatment with NT-3 (Fig. $8 A, B$ ). Knockdown of DISC1 resulted in the reduction of phosphorylated ERK-1/2 at growth cones but not at cell bodies when treated with NT-3 (Fig. $8 A, B$ ). We further investigated whether the expression of DISC1-EGFP instead of EGFP alone could rescue the decrease of ERK-1/2 phosphorylation levels induced by DISC1 knockdown. In the 

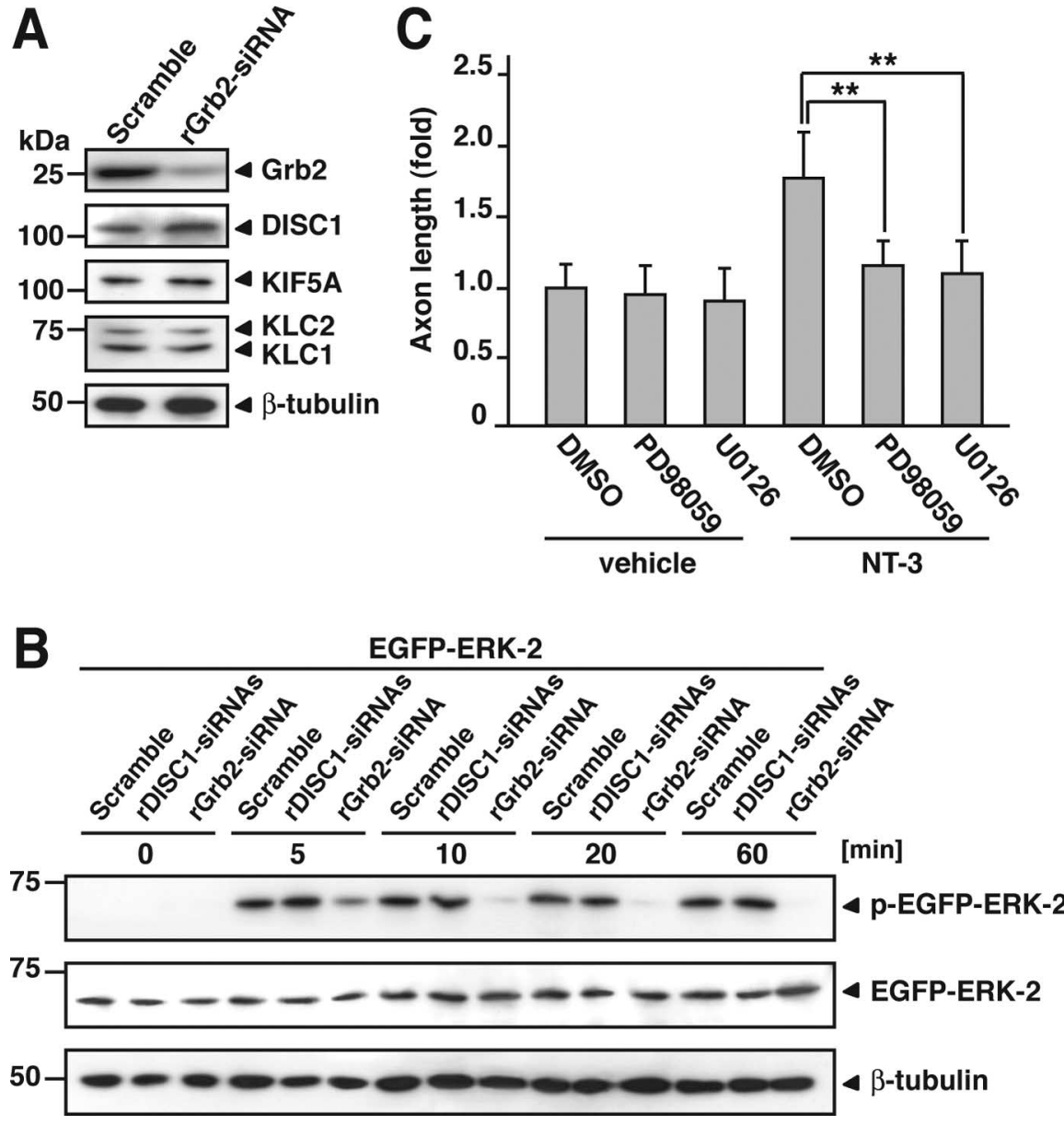

Figure 7. Requirement of Grb2 in ERK phosphorylation during stimulation by neurotrophins. A, PC12 cells were transfected with scramble or rGrb2-siRNA. Cells were cultured and differentiated with NGF for $48 \mathrm{~h}$ after transfection and subjected to immunoblot analysis with antibodies against Grb2, DISC1, KIF5A, KLCs, and $\beta$-tubulin. $\boldsymbol{B}$, Cotransfection of the plasmid encoding EGFP-ERK-2 and scramble, rDISC1-siRNAs, or rGrb2-siRNA was performed in PC12 cells. After culture in medium without serum for $24 \mathrm{~h}$, cells were treated with vehicle or $100 \mathrm{ng} / \mathrm{ml}$ NGF for indicated times. Cells were collected and lysed with SDS sample buffer. Immunoblot analysis was performed with antibodies against phospho-ERK-1/2, GFP, and $\beta$-tubulin. Data are representative of three independent experiments. $C$, Hippocampal neurons were transfected with the expression plasmid encoding EGFP at DIV1. Neurons were then cultured for $2 \mathrm{~d}$ in the medium with vehicle or $100 \mathrm{ng} / \mathrm{mI} \mathrm{NT}-3$ and also with DMSO, PD98059 $(20 \mu \mathrm{M})$, or U0126 $(1 \mu \mathrm{M})$. Cells were fixed at DIV3, followed by immunostaining with anti-GFP antibody. The length of the axon of each EGFP-positive neuron was measured. Numbers of cells used for each calculation are $>50$, and the values shown are means \pm SE of three independent experiments (Student's $t$ test, ${ }^{* *} p<0.01$ ).

neurons expressing DISC1-EGFP, phosphorylation levels of ERK-1/2 at growth cones were restored (Fig. $8 B$ ). These results suggest that DISC1 is required for NT-3-induced ERK phosphorylation at the distal part of axons. We also performed the knockdown of Grb2 in neurons. Although endogenous Grb2 was effectively reduced by the transfection of rGrb2-siRNA in PC12 cells (Fig. 7A), most hippocampal neurons transfected with rGrb2siRNA remained in stage 2 of development (Dotti et al., 1988), even at DIV3 (data not shown), possibly because of the requirement of Ras activity in axon formation in cultured hippocampal neurons (Yoshimura et al., 2006). Thus, we could not determine the direct functions of Grb2 in cultured hippocampal neurons by knockdown of Grb2.

We further examined the functions of DISC1 in axon elongation. Hippocampal neurons were cotransfected with rDISC1siRNAs and the expression plasmid encoding EGFP alone, DISC1-EGFP, or DISC1-2A-EGFP. As we reported in the accompanying paper (Taya et al., 2006), knockdown of DISC1 resulted in shorter axons (Fig. 9A). Defect of the axonal elongation was rescued by the expression of both DISC1-2A as well as wild-type DISC1 (Fig. $9 A$ ), suggesting that the interaction of DISC1 with Grb2 is not required for axon elongation under these conditions. When neurons were cultured with NT-3 for $48 \mathrm{~h}$ after transfection, the lengths of the axons of scramble siRNA-transfected neurons were increased $\sim 1.6$-fold (data not shown) compared with those that were cultured in the absence of NT-3. Knockdown of DISC1 also resulted in the defect of axonal elongation (Fig. 9B). In this condition, the expression of wild-type DISC1 but not DISC1-2A rescued the axonal elongation defect in DISC1 knockdown neurons (Fig. 9B). Defective accumulation of Grb2 at the distal part of axons, conversely, was rescued only by wild-type DISC1 in the absence or presence of NT-3 in a culture medium (Fig. $4 A, B$ ) (data not shown). These results suggest that the association of Grb2 with DISC1 is required for the NT-3-induced axon elongation.

We next examined whether the defect of the axon elongation induced by DISC1 knockdown could be rescued by the overexpression of Grb2. The immunoreactivity of EGFP-Grb2 was observed in the entire neuron, including axon shafts and growth cones (Fig. 6) (data not shown). The expression of EGFP-Grb2 did not significantly alter the length of axons when cultured in the absence of NT-3 (Fig. 9C). Conversely, EGFP-Grb2 partially restored the axon growth defect by DISC1 knockdown in the presence of NT-3 (Fig. 9D). Although we could not show the proper reason for these results, expressed EGFPGrb2 localized at the distal part of axons may compensate for the loss of endogenous Grb2 accumulation by the knockdown of DISC1. Together, these results suggest that the proper localization of Grb2, which is mediated by the DISC1/Kinesin-1 complex, is required for NT-3-induced ERK activation at distal axons and for axon elongation.

\section{Discussion}

Regulation of Grb2 transport by the DISC1/Kinesin-1 complex

In the accompanying paper (Taya et al., 2006), we showed that DISC1 regulates the localization of NUDEL/LIS1/14-3-3 $\varepsilon$ complex in Kinesin-1-dependent manner. In this study, we have shown that knockdown of DISC1 results in the loss of Grb2 accumulation at the distal part of axons (Figs. $3 B, C, 4 A, B$ ). Similarly, the functional blocking of Kinesin-1 by KLC knockdown or expression of the KIF5A-dominant-negative mutant impaired Grb2 accumulation at the distal parts of axons (Fig. 5B-F). Together, these results strongly suggest that DISC1 functions as a cargo receptor between Kinesin-1 and the DISC1-binding partners, such as NUDEL/LIS1/14-3-3e or Grb2. A chromosomal translocation accompanied by a high inheritance of schizophre- 
nia results in the reduction of DISC1 expression (Millar et al., 2005) or the truncation of the DISC1 protein with loss of its C-terminal 257 amino acids (Blackwood et al., 2001). We confirmed that Grb2 as well as NUDEL/LIS1/14-3-3 $\varepsilon$ did not interact with the truncated form of DISC1 (amino acids 1-597) (data not shown) (Taya et al., 2006). We also confirmed that the expression of the truncated form of DISC1 resulted in the delocalization of Grb2 as well as $14-3-3 \varepsilon$ from the distal part of axons (data not shown) (Taya et al., 2006). Thus, the reduction of DISC1 expression or the truncation of DISC1 by the chromosomal translocation possibly impairs the accumulation of Grb2 or NUDEL/LIS1/14-3-3 $\varepsilon$ at the distal part of axons.

\section{Functions of DISC1 and Grb2 in neurons}

In this study, we reported that Grb2 is crucial for ERK phosphorylation during stimulation with neurotrophin and that the activation of the Ras-ERK pathway is necessary for the NT-3-induced axon elongation in cultured hippocampal neurons (Fig. $7 B, C$ ). We also showed that DISC1 is necessary for ERK phosphorylation at the distal part of axons and for NT3 -induced axon elongation (Figs. 8A, $B$, $9 A, B)$. DISC1 itself, conversely, does not seem to be directly involved in the ERK activation pathway (Fig. 7B). Knockdown of DISC1 in cultured hippocampal neurons, however, resulted in reduced axon elongation in the presence or absence of NT-3 (Fig. 9A,B) (Taya et al., 2006). On the basis of these results, we propose two different roles of DISC1 in axon elongation. As we showed in the accompanying paper (Taya et al., 2006), DISC1 transports the NUDEL/LIS1/14-3-3 $\varepsilon$ complex to the distal part of axons via Kinesin-1. LIS1 is known as a member of the +tips, the molecules that accumulate at the plus ends of growing microtubules (Galjart, 2005), and is required for axon elongation (Kholmanskikh et al., 2003). It was also shown in the accompanying paper that the knockdown of NUDEL or LIS1 resulted in defective axon elongation in cultured hippocampal neurons (Taya et al., 2006). Together, NUDEL/LIS1 at the distal part of axons, transported by DISC1, seems to be required for axon elongation. The accumulated Grb2 in the distal part of axons, conversely, is specifically required for ERK phosphorylation after NT-3 stimulation, which leads to the NT-3induced axon elongation.

Grb2 is known to function at the downstream of other receptor tyrosine kinases as well as Trk receptors. Neuregulin, a possible risk factor for schizophrenia, contains an EGF-like domain and binds to its specific receptor tyrosine kinases, ErbB2, ErbB3,

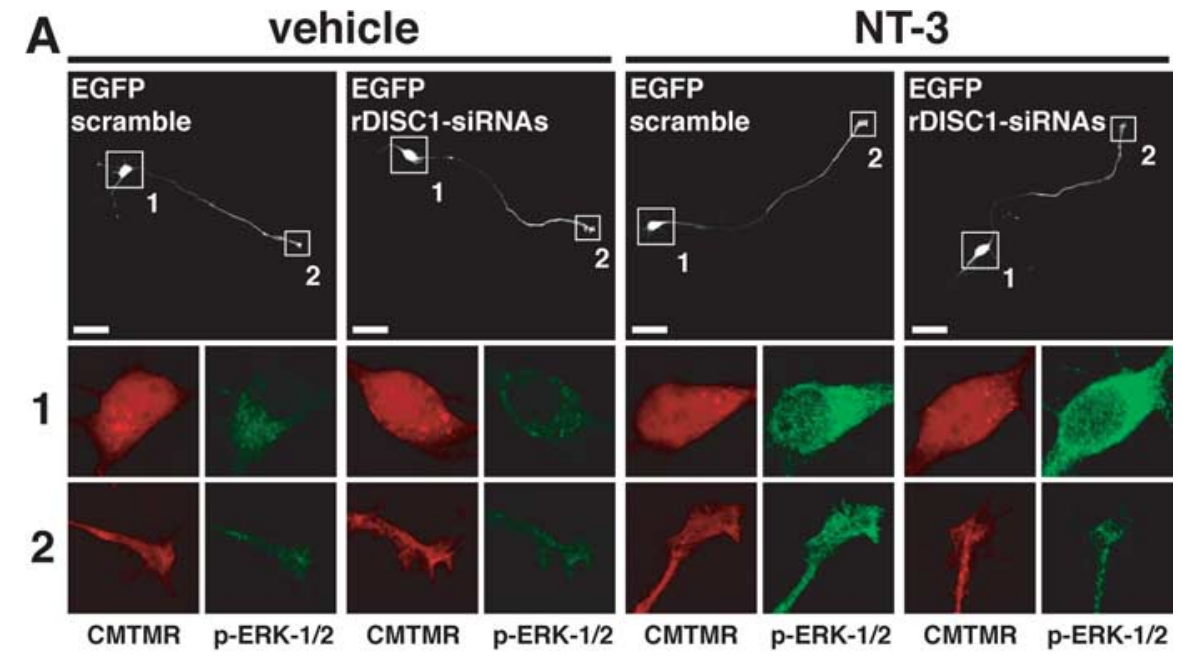

B <Cell bodies>

$<$ Growth cones>
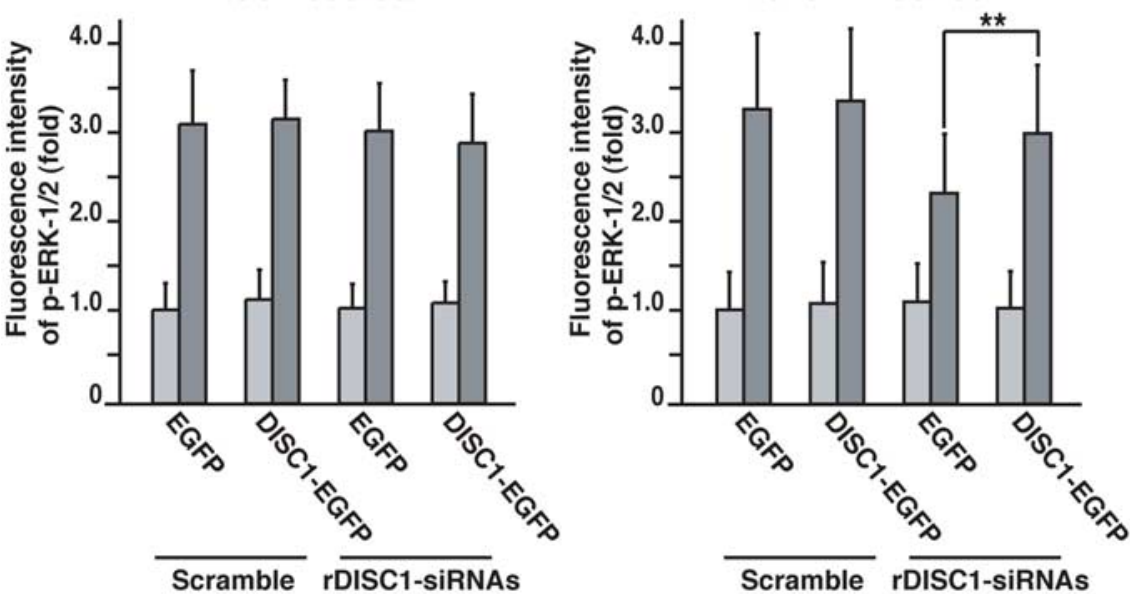

vehicle

NT-3

Figure 8. Requirement of DISC1 in the phosphorylation of ERK-1/2. A, Hippocampal neurons were cotransfected with the expression plasmid encoding EGFP and scramble or rDISC1-siRNAs at DIV0. Neurons were cultured for $3 \mathrm{~d}$ and then treated with vehicle or NT-3 $(100 \mathrm{ng} / \mathrm{ml})$ for $30 \mathrm{~min}$. Cells were fixed and immunostained with antibodies against GFP and phospho-ERK-1/2. Cells were also stained with the cytoplasmic fluorescent probe CMTMR before fixation. Enlarged images of the cell bodies (1) and growth cones (2) of each EGFP-positive neuron are shown. Scale bars, $20 \mu \mathrm{m}$. B, Hippocampal neurons were cotransfected with the expression vector encoding EGFP or EGFP-DISC1 and scramble or rDISC1-siRNAs at DIV0. Neurons were cultured for $3 \mathrm{~d}$ and then treated with vehicle or NT-3 (100 ng/ml) for 30 min. Cells were fixed and immunostained with antibodies against GFP and phospho-ERK-1/2. The fluorescence intensity of immunostained phospho-ERK-1/2 at cell bodies and growth cones were assessed (see Materials and Methods). Numbers of cells used for each calculation are $>50$, and the values shown are means $\pm S E$ of three independent experiments (Student's $t$ test, ${ }^{* *} p<0.01$ ).

and ErbB4 (Falls, 2003). Grb2 directly or indirectly associates with ErbBs in a ligand-dependent manner and possibly mediates Ras-ERK activation (Falls, 2003). ErbB4 is dominantly localized in postsynapse areas in cortical neurons. Grb2 as well as ErbB4 are recruited to neuronal lipid raft by neuregulin stimulation ( $\mathrm{Ma}$ et al., 2003). The signals downstream of Trk receptors are involved not only in axon elongation but also in modulation of neurotransmission. TrkB exists in both presynapses and postsynapses and is involved in synaptic plasticity in hippocampal neurons (Poo, 2001). It was also reported that ERK-1/2 activation by $\mathrm{BDNF}$ is involved in spine formation in dendrites (Alonso et al., 2004). Although the localization of Grb2 in matured neurons is 

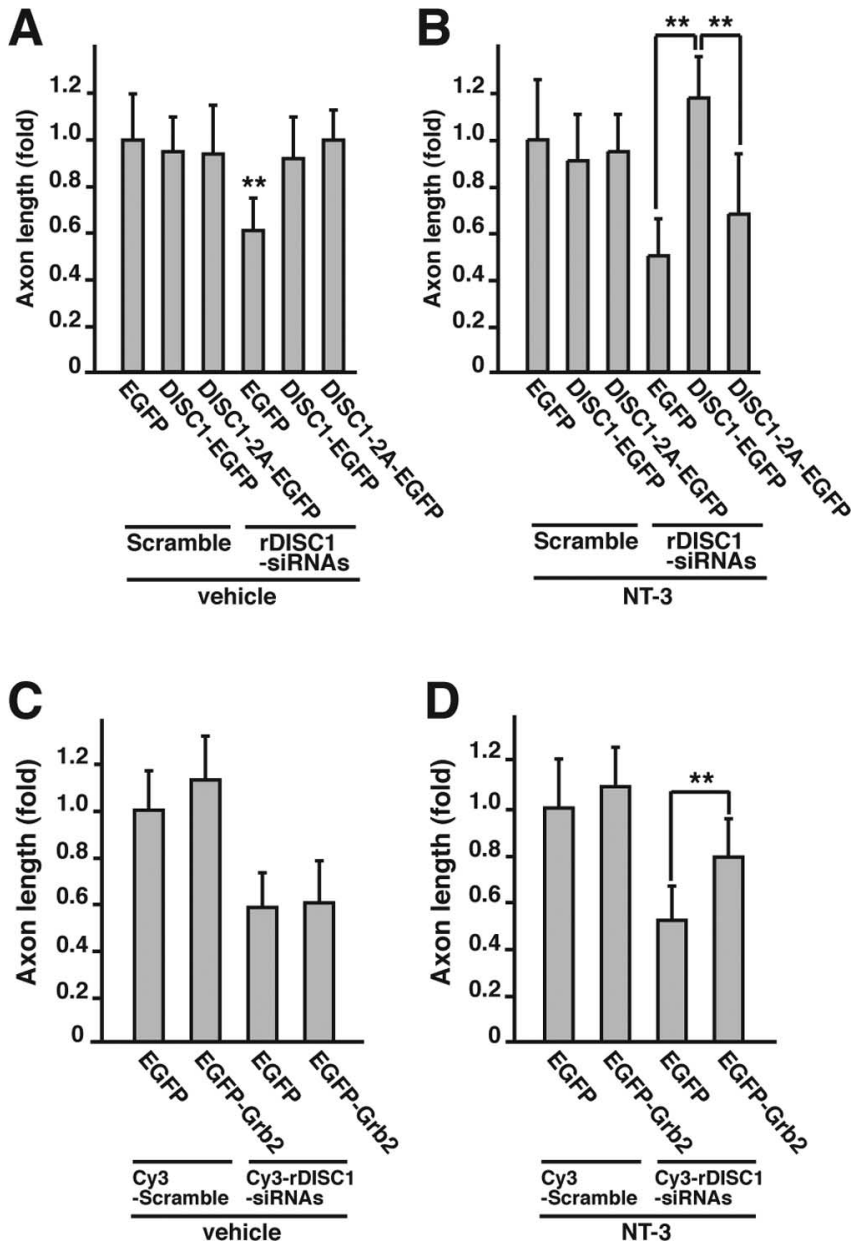

Figure 9. Requirement of DISC1 in NT-3-induced axon elongation. $A, B$, Hippocampal neurons were cotransfected with scramble or rDISC1-siRNAs and expression plasmid encoding EGFP, DISC1-EGFP, or DISC1-2A-EGFP at DIV1. After transfection, neurons were cultured with growth medium $(\boldsymbol{A})$ or with medium containing $100 \mathrm{ng} / \mathrm{ml} \mathrm{NT}-3(\boldsymbol{B})$ and then fixed at DIV3. The transfected neurons were visualized by staining of EGFP. The length of the axon of each EGFPpositive neuron was measured. Numbers of cells used for each calculation are $>50$, and the values shown are means $\pm S E$ of three independent experiments (Student's $t$ test, ${ }^{* *} p<0.01$ ). C, D, Hippocampal neurons were transfected with the expression plasmid encoding EGFP-GST or EGFP-Grb2 at DIV1 and the transfected with Cy3-scramble or Cy3-rDISC1-siRNAs at DIV2. Neurons were then cultured with control growth medium $(C)$ or medium containing $100 \mathrm{ng} / \mathrm{ml}$ NT-3 (D) for $48 \mathrm{~h}$. Transfected cells were identified by the staining of EGFP and Cy3 fluorescence. Length of the axon of each EGFP- and Cy3-positive neuron was measured. Numbers of cells used for each calculation are $>50$, and the values shown are means \pm SE of three independent experiments (Student's $t$ test, ${ }^{* *} p<0.01$ ).

not well understood, it is possible that Grb2 mediates the activation of the Ras-ERK pathway downstream of ErbBs or Trk receptors and is thereby involved in the modulation of spine formation or neurotransmission in synapses.

\section{Neurotrophins and the pathogenesis of schizophrenia}

Neurotrophins regulate cell survival, neuronal differentiation, axon and dendrite growth, and targeting (Huang and Reichardt, 2001, 2003; Chao, 2003). Because most of our knowledge of the neurotrophins has been obtained from assays using peripheral nervous system or neuronal cell lines, such as PC12 cells, the role of neurotrophins in vivo, especially in the CNS, remains essentially unknown. Studies in conditional NT-3 knock-out mice revealed that NT-3 is required for the proper thalamocortical projections (Ma et al., 2002). Experiments using slice cultures demonstrated that $\mathrm{BDNF}$ and NT-3 regulate dendritic growth in cortex (McAllister et al., 1997). Notably, some reports suggested the involvement of NT-3 itself in schizophrenia (Durany et al., 2001; Lin and Tsai, 2004). In this study, we showed that the knockdown of DISC1 results in the defect of NT-3-induced axon elongation (Fig. 9A,B). These findings raise a possibility that the dysfunctions of DISC1 lead neurons to the decreased responses to neurotrophins such as NT-3, thereby resulting in defective neuronal development. A recent study reported that a single nucleotide polymorphism (SNP) accompanied with an amino acid change in DISC1 gene affects hippocampal volume and function (Callicott et al., 2005). It is worthwhile examining whether this amino acid change affects the interaction of DISC1 with the partner proteins.

It is now widely accepted that the pathogenesis of schizophrenia is related to neuronal development (Weinberger, 1987). Brains of schizophrenic patients are often accompanied by morphological alternations, possibly attributable to the reduction of the numbers of neuronal cells and neuritic processes (Harrison, 1999; Lewis and Levitt, 2002). Dysfunctions of DISC1 by the chromosomal translocation or SNPs may therefore result in defective responses of the CNS neurons to neurotrophins such as NT-3, which may account for the pathogenesis of schizophrenia.

\section{References}

Alonso M, Medina JH, Pozzo-Miller L (2004) ERK1/2 activation is necessary for BDNF to increase dendritic spine density in hippocampal CA1 pyramidal neurons. Learn Mem 11:172-178.

Blackwood DH, Fordyce A, Walker MT, St. Clair DM, Porteous DJ, Muir WJ (2001) Schizophrenia and affective disorders-cosegregation with a translocation at chromosome 1q42 that directly disrupts brain-expressed genes: clinical and P300 findings in a family. Am J Hum Genet 69:428-433.

Borrello MG, Pelicci G, Arighi E, De Filippis L, Greco A, Bongarzone I, Rizzetti M, Pelicci PG, Pierotti MA (1994) The oncogenic versions of the Ret and Trk tyrosine kinases bind Shc and Grb2 adaptor proteins. Oncogene 9:1661-1668.

Buday L (1999) Membrane-targeting of signalling molecules by SH2/SH3 domain-containing adaptor proteins. Biochim Biophys Acta 1422: 187-204.

Callicott JH, Straub RE, Pezawas L, Egan MF, Mattay VS, Hariri AR, Verchinski BA, Meyer-Lindenberg A, Balkissoon R, Kolachana B, Goldberg TE, Weinberger DR (2005) Variation in DISC1 affects hippocampal structure and function and increases risk for schizophrenia. Proc Natl Acad Sci USA 102:8627-8632.

Campenot RB, Maclnnis BL (2004) Retrograde transport of neurotrophins: fact and function. J Neurobiol 58:217-229.

Chao MV (2003) Neurotrophins and their receptors: a convergence point for many signalling pathways. Nat Rev Neurosci 4:299-309.

Craddock N, O’Donovan MC, Owen MJ (2005) The genetics of schizophrenia and bipolar disorder: dissecting psychosis. J Med Genet 42:193-204.

Craig AM, Banker GA (1994) Neuronal polarity. Annu Rev Neurosci $17: 267-310$.

Dent EW, Gertler FB (2003) Cytoskeletal dynamics and transport in growth cone motility and axon guidance. Neuron 40:209-227.

Dotti CG, Sullivan CA, Banker GA (1988) The establishment of polarity by hippocampal neurons in culture. J Neurosci 8:1454-1468.

Durany N, Michel T, Zochling R, Boissl KW, Cruz-Sanchez FF, Riederer P, Thome J (2001) Brain-derived neurotrophic factor and neurotrophin 3 in schizophrenic psychoses. Schizophr Res 52:79-86.

Ekelund J, Hennah W, Hiekkalinna T, Parker A, Meyer J, Lonnqvist J, Peltonen L (2004) Replication of 1q42 linkage in Finnish schizophrenia pedigrees. Mol Psychiatry 9:1037-1041.

Errede B, Cade RM, Yashar BM, Kamada Y, Levin DE, Irie K, Matsumoto K (1995) Dynamics and organization of MAP kinase signal pathways. Mol Reprod Dev 42:477-485.

Falls DL (2003) Neuregulins: functions, forms, and signaling strategies. Exp Cell Res 284:14-30.

Galbraith JA, Reese TS, Schlief ML, Gallant PE (1999) Slow transport of 
unpolymerized tubulin and polymerized neurofilament in the squid giant axon. Proc Natl Acad Sci USA 96:11589-11594.

Galjart N (2005) CLIPs and CLASPs and cellular dynamics. Nat Rev Mol Cell Biol 6:487-498.

Guzik BW, Goldstein LS (2004) Microtubule-dependent transport in neurons: steps towards an understanding of regulation, function and dysfunction. Curr Opin Cell Biol 16:443-450.

Harrison PJ (1999) The neuropathology of schizophrenia: a critical review of the data and their interpretation. Brain 122:593-624.

Harrison PJ, Weinberger DR (2005) Schizophrenia genes, gene expression, and neuropathology: on the matter of their convergence. Mol Psychiatry 10:40-68.

Hennah W, Varilo T, Kestila M, Paunio T, Arajarvi R, Haukka J, Parker A, Martin R, Levitzky S, Partonen T, Ekelund J (2003) Haplotype transmission analysis provides evidence of association for DISC1 to schizophrenia and suggests sex-dependent effects. Hum Mol Genet 12:3151-3159.

Hirokawa N, Takemura R (2004) Kinesin superfamily proteins and their various functions and dynamics. Exp Cell Res 301:50-59.

Hodgkinson CA, Goldman D, Jaeger J, Persaud S, Kane JM, Lipsky RH, Malhotra AK (2004) Disrupted in schizophrenia 1 (DISC1): association with schizophrenia, schizoaffective disorder, and bipolar disorder. Am J Hum Genet 75:862-872.

Huang EJ, Reichardt LF (2001) Neurotrophins: roles in neuronal development and function. Annu Rev Neurosci 24:677-736.

Huang EJ, Reichardt LF (2003) Trk receptors: roles in neuronal signal transduction. Annu Rev Biochem 72:609-642.

Iida N, Namikawa K, Kiyama H, Ueno H, Nakamura S, Hattori S (2001) Requirement of Ras for the activation of mitogen-activated protein kinase by calcium influx, cAMP, and neurotrophin in hippocampal neurons. J Neurosci 21:6459-6466.

Inagaki N, Chihara K, Arimura N, Menager C, Kawano Y, Matsuo N, Nishimura T, Amano M, Kaibuchi K (2001) CRMP-2 induces axons in cultured hippocampal neurons. Nat Neurosci 4:781-782.

Ip NY, Li Y, Yancopoulos GD, Lindsay RM (1993) Cultured hippocampal neurons show responses to BDNF, NT-3, and NT-4, but not NGF. J Neurosci 13:3394-3405.

Jiang X, Huang F, Marusyk A, Sorkin A (2003) Grb2 regulates internalization of EGF receptors through clathrin-coated pits. Mol Biol Cell 14:858-870.

Kholmanskikh SS, Dobrin JS, Wynshaw-Boris A, Letourneau PC, Ross ME (2003) Disregulated RhoGTPases and actin cytoskeleton contribute to the migration defect in Lis1-deficient neurons. J Neurosci 23:8673-8681.

Kimura T, Watanabe H, Iwamatsu A, Kaibuchi K (2005) Tubulin and CRMP-2 complex is transported via Kinesin-1. J Neurochem 93:1371-1382.

Kouhara H, Hadari YR, Spivak-Kroizman T, Schilling J, Bar-Sagi D, Lax I, Schlessinger J (1997) A lipid-anchored Grb2-binding protein that links FGF-receptor activation to the Ras/MAPK signaling pathway. Cell 89:693-702.

Labbele C, Leclerc N (2000) Exogenous BDNF, NT-3 and NT-4 differentially regulate neurite outgrowth in cultured hippocampal neurons. Brain Res Dev Brain Res 123:1-11.

Lewis DA, Levitt P (2002) Schizophrenia as a disorder of neurodevelopment. Annu Rev Neurosci 25:409-432.

Lin PY, Tsai G (2004) Meta-analyses of the association between genetic polymorphisms of neurotrophic factors and schizophrenia. Schizophr Res 71:353-360.

Lowenstein EJ, Daly RJ, Batzer AG, Li W, Margolis B, Lammers R, Ullrich A, Skolnik EY, Bar-Sagi D, Schlessinger J (1992) The SH2 and SH3 domain-containing protein GRB2 links receptor tyrosine kinases to Ras signaling. Cell 70:431-442.

Ma L, Harada T, Harada C, Romero M, Hebert JM, McConnell SK, Parada LF (2002) Neurotrophin-3 is required for appropriate establishment of thalamocortical connections. Neuron 36:623-634.

Ma L, Huang YZ, Pitcher GM, Valtschanoff JG, Ma YH, Feng LY, Lu B, Xiong WC, Salter MW, Weinberg RJ, Mei L (2003) Ligand-dependent recruit- ment of the ErbB4 signaling complex into neuronal lipid rafts. J Neurosci 23:3164-3175.

MacDonald JI, Gryz EA, Kubu CJ, Verdi JM, Meakin SO (2000) Direct binding of the signaling adapter protein Grb2 to the activation loop tyrosines on the nerve growth factor receptor tyrosine kinase, TrkA. J Biol Chem 275:18225-18233.

Markus A, Zhong J, Snider WD (2002) Raf and Akt mediate distinct aspects of sensory axon growth. Neuron 35:65-76.

McAllister AK, Katz LC, Lo DC (1997) Opposing roles for endogenous BDNF and NT-3 in regulating cortical dendritic growth. Neuron 18:767-778.

Millar JK, Wilson-Annan JC, Anderson S, Christie S, Taylor MS, Semple CA, Devon RS, Clair DM, Muir WJ, Blackwood DH, Porteous DJ (2000) Disruption of two novel genes by a translocation co-segregating with schizophrenia. Hum Mol Genet 9:1415-1423.

Millar JK, Pickard BS, Mackie S, James R, Christie S, Buchanan SR, Malloy MP, Chubb JE, Huston E, Baillie GS, Thomson PA, Hill EV, Brandon NJ, Rain JC, Camargo LM, Whiting PJ, Houslay MD, Blackwood DH, Muir WJ, Porteous DJ (2005) DISC1 and PDE4B are interacting genetic factors in schizophrenia that regulate cAMP signaling. Science 310:1187-1191.

Morris JA, Kandpal G, Ma L, Austin CP (2003) DISC1 (Disrupted-inSchizophrenia 1) is a centrosome-associated protein that interacts with MAP1A, MIPT3, ATF4/5 and NUDEL: regulation and loss of interaction with mutation. Hum Mol Genet 12:1591-1608.

Nakata T, Hirokawa N (2003) Microtubules provide directional cues for polarized axonal transport through interaction with kinesin motor head. J Cell Biol 162:1045-1055.

Nimnual AS, Yatsula BA, Bar-Sagi D (1998) Coupling of Ras and Rac guanosine triphosphatases through the Ras exchanger Sos. Science 279:560-563.

Nishimura T, Kato K, Yamaguchi T, Fukata Y, Ohno S, Kaibuchi K (2004) Role of the PAR-3-KIF3 complex in the establishment of neuronal polarity. Nat Cell Biol 6:328-334.

Owen MJ, Williams NM, O'Donovan MC (2004) The molecular genetics of schizophrenia: new findings promise new insights. Mol Psychiatry 9:14-27.

Ozeki Y, Tomoda T, Kleiderlein J, Kamiya A, Bord L, Fujii K, Okawa M, Yamada N, Hatten ME, Snyder SH, Ross CA, Sawa A (2003) Disruptedin-Schizophrenia-1 (DISC-1): mutant truncation prevents binding to NudE-like (NUDEL) and inhibits neurite outgrowth. Proc Natl Acad Sci USA 100:289-294.

Pawson T (1993) Protein modules and signaling networks. Nature 373: 573-580.

Poo MM (2001) Neurotrophins as synaptic modulators. Nat Rev Neurosci 2:24-32.

Qian X, Riccio A, Zhang Y, Ginty DD (1998) Identification and characterization of novel substrates of Trk receptors in developing neurons. Neuron 21:1017-1029.

Rozakis-Adcock M, Fernley R, Wade J, Pawson T, Bowtell D (1993) The $\mathrm{SH} 2$ and $\mathrm{SH} 3$ domains of mammalian Grb2 couple the EGF receptor to the Ras activator mSos1. Nature 363:83-85.

Takenawa T, Miki H, Matuoka K (1998) Signaling through Grb2/Ashcontrol of the Ras pathway and cytoskeleton. Curr Top Microbiol Immunol 228:325-342.

Taya S, Shinoda T, Nagai K, Tuboi D, Kuroda K, Hikita T, Asaki J, Shimizu M, Hirotune S, Iwamatu A, Kaibuchi K (2006) Disrupted-inSchizophrenia-1 is required for axon elongation by regulating NUDEL/ LIS1/14-3-3e complex transport. J Neurosci 26:15-26.

Vale RD (2003) The molecular motor toolbox for intracellular transport. Cell 112:467-480.

Weinberger DR (1987) Implications of normal brain development for the pathogenesis of schizophrenia. Arch Gen Psychiatry 44:660-669.

Yoshimura T, Kawano Y, Arimura N, Kawabata S, Kikuchi A, KaibuchiK (2005) GSK-3 regulates phosphorylation of CRMP-2 and neuronal polarity. Cell 120:137-149.

Yoshimura T, Arimura N, Kawano Y, Kawabata S, Wang S, Kaibuchi K (2006) Ras regulates neuronal polarity via the PI3-kinase/Akt/GSK3beta/CRMP-2 pathway. Biochem Biophys Res Commun 340:62-68. 